\title{
Apoptotic death of photoreceptors in the streptozotocin-induced diabetic rat retina
}

\author{
S.-H. Park, J.-W. Park, S.-J. Park, K.-Y. Kim, J.-W. Chung, M.-H. Chun, S.-J. Oh \\ Department of Anatomy, College of Medicine, The Catholic University of Korea, Socho-gu, Seoul, Korea
}

\section{Abstract}

Aims/hypothesis. Neurodegenerative changes in the diabetic retina occurring before diabetic retinopathy could be inevitable by the altered energy (glucose) metabolism, in the sense that dynamic image-processing activity of the retinal neurons is exclusively dependent on glucose. We therefore investigated the morphological changes in the neural retina, including neuronal cell death, of a streptozotocin-induced model of diabetes.

Methods. Streptozotocin was intravenously injected. Rats were maintained hyperglycaemic without insulin treatment for 1 week and $4,8,12$, and 24 weeks, respectively. Diabetic retinas were processed for histology, electron microscopy, and immunohistochemistry using the TUNEL method.

Results. A slight reduction in the thickness of the inner retina was observed throughout the diabetic retinas and a remarkable reduction was seen in the outer nuclear layer 24 weeks after the onset of diabetes.
The post-synaptic processes of horizontal cells in the deep invaginations of the photoreceptors showed degeneration changes from 1 week onwards. A few necrotic ganglion cells were observed after 4 weeks. At 12 weeks, some amacrine cells and a few horizontal cells showed necrotic features. Three to seven cellular layers in the outer nuclear layer and nerve terminals, rolled by the fine processes of the Müller cells near the somata of the degenerated ganglion cells, were apparent at 24 weeks. Apoptosis appeared in a few photoreceptor cells at 4 weeks, and the number of apoptotic photoreceptors increased thereafter.

Conclusion/interpretation. These findings suggest that the visual loss associated with diabetic retinopathy could be attributed to an early phase of substantial photoreceptor loss, in addition to later microangiopathy. [Diabetologia (2003) 46:1260-1268]

Keywords Apoptosis, photoreceptors, retina, neuronal degeneration, ultrastructure, TUNEL, diabetes.
Diabetic retinopathy is the most common complication of diabetes and a leading cause of vision loss.

Received: 3 December 2002 / Revised: 10 February 2003

Published online: 31 July 2003

(C) Springer-Verlag 2003

Corresponding author: Dr. S.-J. Oh, Department of Anatomy, College of Medicine, The Catholic University of Korea, 505 Banpo-dong, Socho-gu, Seoul 137-701, Korea

E-mail: sujaoh@catholic.ac.kr

Abbreviations: TUNEL, Terminal deoxynucleotidyl transferase-mediated dUTP nick end labelling; GCL, ganglion cell layer; IPL, inner plexiform layer; INL, inner nuclear layer; OPL, outer plexiform layer; ONL, outer nuclear layer.
Many of the studies addressing diabetic retinopathy have focused on the clinically well-recognized feature of microangiopathy and its underlying mechanisms to develop protective strategies directed against exacerbated angiogenesis and subsequent vision loss. Hyperglycaemia induced at the initiation of diabetes causes tissue damage by several major mechanisms, including increased traffic through the polyol pathway, increased de novo synthesis of diacylglycerol, altered intracellular redox state, and AGE formation $[1,2]$. AGE, together with reactive oxygen species, attacks the endothelial cells of the blood vessels, and could contribute to the breakdown of the diabetic blood-retinal barrier and the adher- 
ence of leucocytes, by losing such characteristics as the site of the blood-tissue barrier and the nonthrombogenic surface for blood flow, thereby contributing to the development of microangiopathy and attendant unregulated angiogenesis [3].

The retina, as a part of the central nervous system, uses glucose as an exclusive energy source for dynamic activity such as for capturing images and for primary visual processing. The glucose used for retinal neuronal activity is transported from the blood by two vasculatures: by the deep and superficial layers of the retina's own blood vessels, and by the capillaries of the choroids [4, 5]. Radial Müller cells, as an additional glial component of the blood-retinal barrier, are present spanning the whole retinal thickness and wrapping the apposed neurons with their thin cytoplasmic processes $[3,6]$. Therefore, systemically impaired glucose metabolism causes dysfunction in the neural retina soon after the onset of diabetes, whereas microangiopathy sets in after an extended period of long-standing hyperglycaemia.

Abnormalities of the neural retina have been reported in experimental and human diabetes. Loss of colour sensitivity [7] and contrast sensitivity [8] are early signs of neural retinal dysfunction that occur after only 2 years of diabetes. Changed metabolism of major inhibitory (GABA) and excitatory (glutamate) neurotransmitters has been documented in diabetic rats and diabetic patients $[9,10]$. The oscillatory potentials of the b-waves on electroretinograms arising from Müller-cell function become altered after only a few years in diabetic patients [11]. Impaired glial reactivity and apoptotic cell death of retinal ganglion cells have also been observed in cases of short-term experimental diabetes and in humans with diabetes $[10,12]$. It is therefore important to characterize the early pathological processes in the diabetic neural retina before the onset of vascular pathology.

The neural retina is composed of diverse neurons, characterized by morphological and biochemical criteria, and numerous neural networks formed through chemical and electrical synapses between these neuronal processes. These neural components are arranged into retinal layers. Retinal neurons are sensitive differentially to a variety of stimuli, depending on the type of injury, and subtypes within one neuronal type also show differential sensitivity. Ganglion cells, for instance, are more sensitive to hypoxic conditions and glutamate excitotoxicity, whereas all neuronal types are damaged by hypoglycaemia [13]. Moreover, retinal ganglion cells respond differentially to excitotoxicity, depending on soma size and retinal eccentricity [14]. In the context of the differential sensitivity of retinal neurons, where and at which level in the visual processing pathway the neuron is located as well as the characteristic of the neuronal environment, could be key factors in determining the sensitivity of a specific neuron to a stimulus.
Taken together, the neurodegenerative changes in the diabetic retina, accessed at the morphological level by electron microscopy and also studied over a given course of time, could provide some insight into the mechanisms underlying the early pathogenesis of the neural retina that leads to diabetic retinopathy.

\section{Materials and methods}

Establishment of the diabetic condition. Male Sprague-Dawley rats ( 8 weeks old; blood glucose concentrations $80-120 \mathrm{mg} / \mathrm{dl}$ ) were used for this study. The diabetic state was induced with a single injection of streptozotocin $(60 \mathrm{mg} / \mathrm{kg}$ body weight in $0.05 \mathrm{~mol} / \mathrm{l}$ sodium citrate buffer, $\mathrm{pH}$ 5.5; Sigma, St. Louis, Mo., USA) via a femoral vein, under anesthesia produced with $4 \%$ chloral hydrate $(1 \mathrm{ml} / 100 \mathrm{~g}$ body weight $)$. To evaluate the optimal induction of diabetes, blood glucose concentrations of the injected animals were checked using a glucometer (Glucotrend, Roche, England) during a fasting state 2 days after the injection. Animals with glucose concentrations above $300 \mathrm{mg} / \mathrm{dl}$ were selected and maintained on a 12-h alternating light to dark cycle. Rats were allowed food and water ad libitum. The experimental term of induced diabetes was set at 1 , $4,8,12$, or 24 weeks, and five animals were assigned to each term group. We used 8-week-old young adult rats as the normal control group. Non-injected rats were also cared for 24 weeks as an age-matched control group to compare the possibility of senile neurodegenerative changes. The treatment of animals conformed to the guidelines of the Catholic Ethics Committee of the Catholic University of Korea, Seoul, in accordance with the Principles of laboratory animals care $(\mathrm{NIH}$ publication no. 85-23, revised 1985).

Tissue preparation. At each experimental time-point, the eyeballs were enucleated from the diabetic rats under anesthesia induced with $16.5 \%$ urethane $(10 \mathrm{mg} / \mathrm{kg}$ body weight) after checking the final blood glucose concentration and body weight. The animals were then killed with an overdose of urethane. The eyeballs were opened with an encircling cut along the ora serrata and the posterior eyecups were immersed in $4 \%$ paraformaldehyde in $0.1 \mathrm{~mol} / 1$ phosphate buffer, pH 7.4 (PB) for $30 \mathrm{~min}$. The retina was dissected from the choroids and the central portion of the superior nasal quadrant was trimmed into small pieces or the whole retina was immersed further in the same fixative. The immersion-fixed retinal tissues were soaked in $30 \%$ sucrose solution in PB and stored frozen for later use.

Morphological observation. Further immersion-fixation of the retinal pieces was done in $2 \%$ paraformaldehyde with $2.5 \%$ glutaraldehyde solution, and then in $1 \%$ osmium tetroxide solution in PB. After being thoroughly rinsed with PB, the pieces were dehydrated in a graded ethanol series, passed through acetone, and embedded in Epon 812 mixture (Polyscience, Warrington, Penn., USA). Samples were polymerized at $60^{\circ} \mathrm{C}$ for 3 days. From these polymerized tissue blocks, $1-\mu \mathrm{m}$-thick semi-thin sections were cut using an ultramicrotome (Reichert-Jung, $\mathrm{Nu}$ ßloch, Germany), then mounted on gelatin-coated glass slides, and stained with $1 \%$ toluidine blue. Next, 70-nm-thick ultra-thin sections were cut from the well-preserved and defined areas of the semi-thin sections identified by light microscopy. Ultra-thin sections were mounted on Formvar (Merck, Darmstadt, Germany)-coated one-hole grids. The ultra-thin sections were double stained with $1 \%$ uranyl acetate and lead citrate, and observed with electron microscopy (JEM 1200B, Jeol, Japan). 
TUNEL staining. To identify apoptotic cell death in the retinal neurons affected by diabetes, the whole retinas were treated with a modified terminal deoxynucleotidyl-transferase-mediated dUTP nick end labelling (TUNEL) method, following the protocol given in the In Situ Cell Detection Kit (Boehringer Mannheim, Mannheim, Germany). To enhance the permeability of the cells, the whole retinas were incubated in $0.2 \%$ Triton $\mathrm{X}-100$ for $5 \mathrm{~min}$ and rinsed in distilled water. Endogenous peroxidase activity was blocked by placing the retinas in $3 \%$ $\mathrm{H}_{2} \mathrm{O}_{2}$ in distilled water for $10 \mathrm{~min}$. The retinas were equilibrated in terminal-transferase labelling buffer (Boehringer Mannheim), and the tailing reaction was done with terminal deoxynulceotide transferase $(0.3$ e.u./ $\mu \mathrm{l})$ and biotinylated deoxyuridine triphosphate $(1 \mathrm{nmol} / \mathrm{l} / \mu \mathrm{l})$ in a humidified chamber for $1 \mathrm{~h}$ at $37^{\circ} \mathrm{C}$. The reaction was terminated by incubation in a solution containing $0.3 \mathrm{~mol} / \mathrm{l} \mathrm{NaCl}$ and $0.03 \mathrm{~mol} / \mathrm{l}$ sodium citrate. The retinas were rinsed in distilled water and treated with $2 \%$ bovine serum albumin in $0.01 \mathrm{~mol} / 1$ phosphate-buffered saline (PBS, $\mathrm{pH}$ 7.4) for $30 \mathrm{~min}$ at room temperature, followed by several rinses in PBS. To visualize the incorporated biotinylated dUTP, the retinas were processed with avidin-biotin-peroxidase complex (ABC, Vector Laboratories, Burlingame, Calif., USA) in PBS for $30 \mathrm{~min}$ at room temperature, and the peroxidase reaction was done with $0.05 \%$ 3,3'-diaminobenzidine$0.01 \% \mathrm{H}_{2} \mathrm{O}_{2}$ in $0.05 \mathrm{~mol} / \mathrm{l}$ Tris- $\mathrm{HCl}$ buffer ( $\mathrm{pH} 7.4$ ) as substrate. The superior halves of the TUNEL stained retinas were mounted with the ganglion cell layer upward, and the inferior halves with the outer nuclear layer upward.

Histological analysis. To analyse the pattern of alterations in the retinal structure at each experimental time-point, the thickness of each retinal layer was measured in at least ten sections from each animal, using the BMI-PLUS image analysis system (Bummi Universe, Ansan, Korea). The reliability of this measurement was ensured by checking one cellular layer of the ganglion cells in the vertical retinal sections at every experimental stage. Using a $40 \times$ objective and a $10 \times$ eyepiece on the microscope, images of each section were captured and then processed. The mean values for the thickness of each retinal layer at each time-point have been represented as means \pm standard deviations.

\section{Results}

The chemically-induced insulin-dependent diabetic condition was properly established in our rats, as

Table 1. Body weight and blood glucose concentration of normal control and diabetic rats

\begin{tabular}{lll}
\hline & $\begin{array}{l}\text { Average of } \\
\text { Body weight } \\
(\mathrm{g})\end{array}$ & $\begin{array}{l}\text { Average of } \\
\text { blood glucose } \\
\text { concentration } \\
(\mathrm{mg} / \mathrm{dl})\end{array}$ \\
\hline Normal group & $205.8 \pm 11.17$ & $110.0 \pm 16.17$ \\
Diabetic group & & \\
1 week & $143.3 \pm 25.17$ & $357.0 \pm 52.50$ \\
4 weeks & $187.0 \pm 36.33$ & $517.0 \pm 51.10$ \\
12 weeks & $162.0 \pm 27.75$ & $509.2 \pm 113.21$ \\
24 weeks & $186.3 \pm 37.72$ & $526.5 \pm 53.24$ \\
\hline
\end{tabular}

Data are given as means \pm standard deviation judged by the high blood glucose concentrations and the remarkable reduction in body weight of the diabetic animals (Table 1).

Histological changes in the diabetic retina. The normal retina has five well-defined layers in light microscopic views of semi-thin sections. These are the ganglion cell layer (GCL), the inner plexiform layer (IPL), the inner nuclear layer (INL), the outer plexiform layer (OPL), and the outer nuclear layer (ONL), in order from the vitreal to scleral sides (Fig. 1A). In the retina of 1-week diabetic rat, the vitreal radial pro-

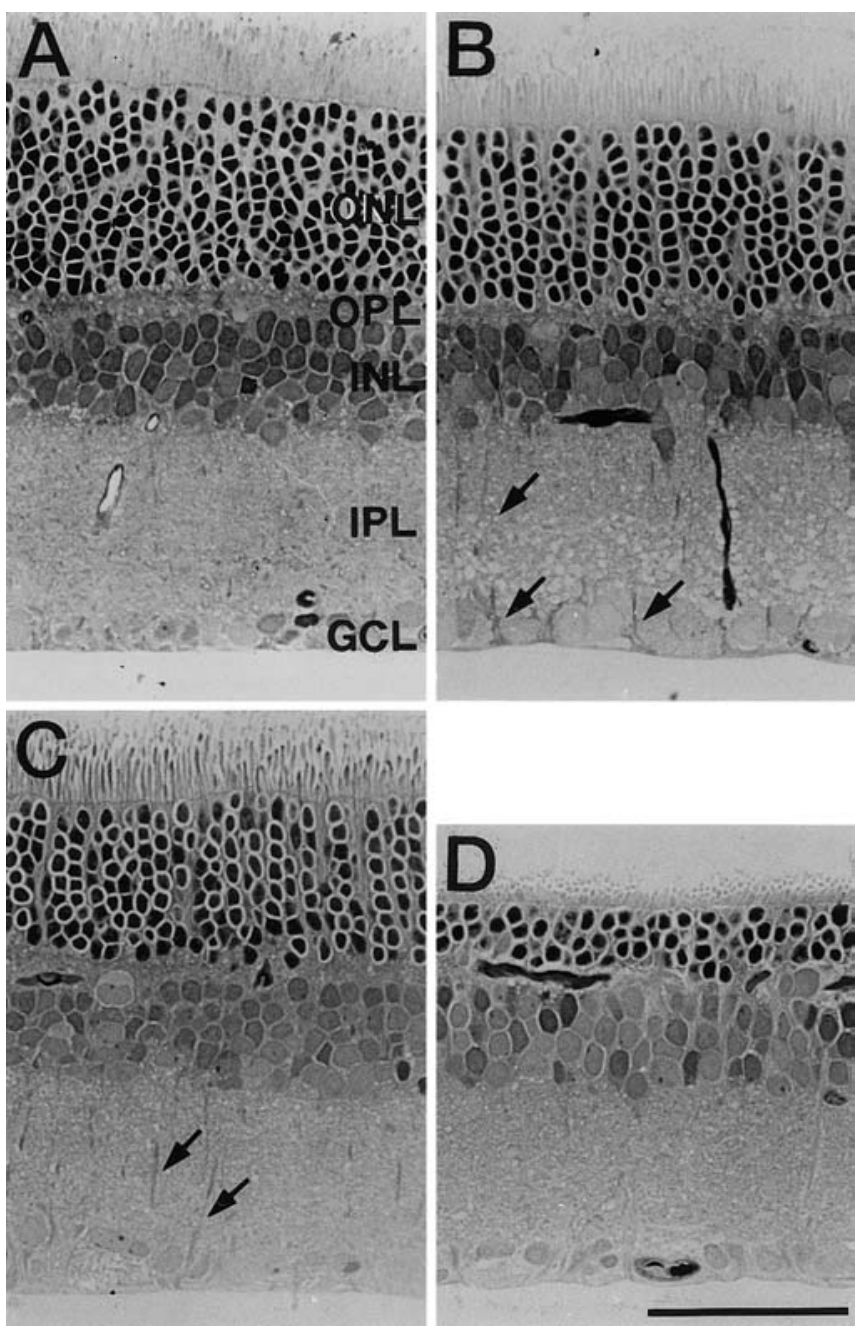

Fig. 1A-D. Light microphotographs of 1- $\mu$ m-thick retinal sections taken from normal rat (A), and rats at 1 week $(\mathbf{B}), 12$ weeks (C), and 24 weeks (D) after the onset of diabetes. In A, the retinal structure is well organized into layers. In $\mathbf{B}$, proximal radial processes of Müller cells (arrows) are clearly visible in the ganglion cell layer (GCL) and the inner plexiform layer (IPL). In $\mathbf{C}$, the photoreceptor cells in the outer nuclear layer (ONL) are separated from each other and are encapsulated within an inter-photoreceptor matrix. Radial processes (arrows) are still present in the GCL and the IPL. In D, the thickness of the retina is remarkably reduced due to the loss of photoreceptor cells. INL, inner nuclear layer; OPL, outer plexiform layer. Scale bar represents $50 \mu \mathrm{m}$ 


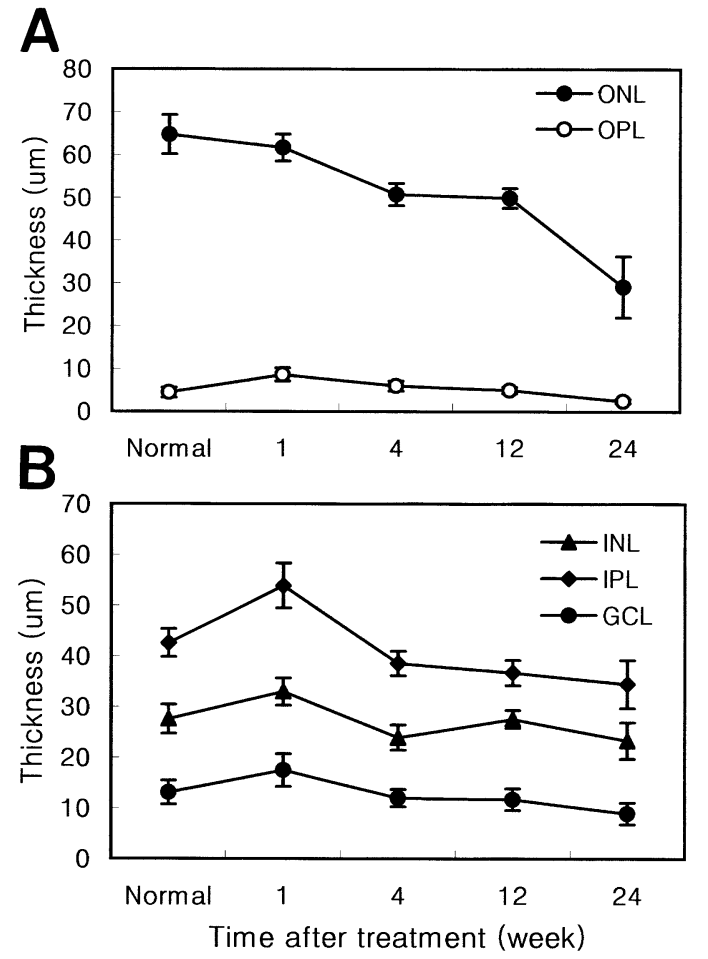

Fig. 2A, B. The graphs show changes in the thickness of each retinal layer of the normal and diabetic retinas. In $\mathbf{A}$, the thickness of the outer nuclear layer (ONL) and of the outer plexiform layer (OPL) in the diabetic retinas is indicated. The line indicating the changes in thickness of the ONL declines gradually up to 12 weeks and then drops suddenly at 24 weeks. In contrast, the line representing the thickness of the OPL remains nearly horizontal. In $\mathbf{B}$, the changes in thickness of the inner nuclear layer (INL), the inner plexiform layer (IPL), and the ganglion cell layer (GCL) at different diabetic stages are shown. Each of the three layers displays a similar pattern at the different experimental time-points, indicating a transient increase at one week and a subsequent slight decrease

cesses of Müller cells stained moderately and became prominent (Fig. 1B). This feature continued into later stages. The cellular arrangement of the ONL in the 12-week diabetic retinas was looser, and the scleral radial processes of Müller cells were clearly visible in between the loosened columns of photoreceptor cells (Fig. 1C). The most remarkable change in the diabetic retinas appeared in the ONL of the 24-week diabetic retina, in which the number of nuclear layers of photoreceptor cells had decreased to 3-7 compared with $10-12$ of the normal young adult retina and 8-12 of the age-matched retina (Fig. 1D).

The changes in thickness of the retinal layers at each diabetic stage were not great in the inner retina, which consists of the GCL, IPL, and INL (Fig. 2B). The thickness of the inner retina increased slightly and temporarily in the 1-week diabetic retinas, and decreased slightly thereafter. In contrast with the slight change in the inner retina, the thickness of the ONL showed an abrupt decrease only at 24 weeks (Fig. 2A). There was little change in the thickness

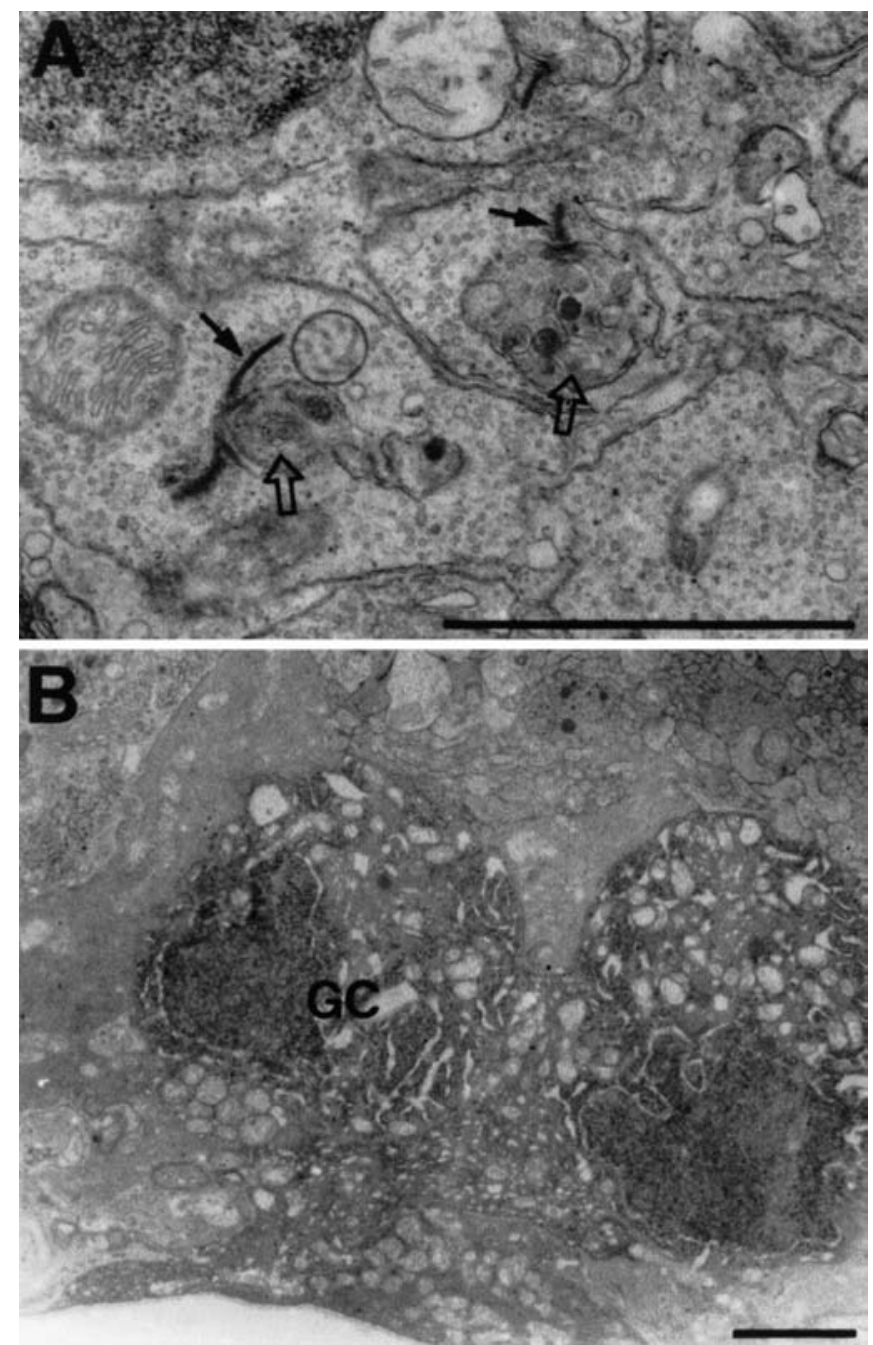

Fig. 3A, B. Electron microphotographs of parts of the rat retina at one week (A) and 4 weeks (B) after the onset of diabetes. Photoreceptor terminals containing ribbon synapses (arrows), and their post-synpatic terminals with degenerated mitochondria (open arrows), were observed in the outer plexiform layer at one week of diabetes. Ganglion cells (GC) showing necrotic profiles appeared at 4 weeks of diabetes. Scale bars represent $2 \mu \mathrm{m}$

of the OPL in the diabetic retinas, although this layer was very thin (less than $10 \mu \mathrm{m})$ in the normal retina.

Pathological changes of neuronal components detected by electron microscopy. The first sign of diabetic influence on the neural retina was detected as myelinated and multi-vesicular features of the mitochondria at one week. Degenerating mitochondria with these features appeared in a few photoreceptor terminals and their post-synaptic processes in the OPL, and in some axon terminals in the IPL near the capillaries (Fig. 3A). The post-synaptic processes of photoreceptor terminals with degenerative profiles were located on the lateral sides in the deep invaginations. Nerve terminals containing degenerating mitochondria were 
frequently observed near the stout radial processes of Müller cells in the IPL by 4 weeks. Necrotic features were also found in a few ganglion cells of the GCL at this time-point, whereas no sign of degeneration appeared in displaced amacrine cells (Fig. 3B). Numerous photoreceptor terminals showed electron-dense cytoplasm and apoptotic nuclei were rarely observed among the photoreceptor cells in the 8-week diabetic retina (Fig. 4). In the 12-week diabetic retina, photoreceptor cells with apoptotic features appeared in the ONL, whereas some ganglion cells in the GCL and some amacrine cells in the INL showed necrotic changes, as containing extraordinarily electron-dense cytoplasmic inclusions (Fig. 5B). Necrotic horizontal cells identified by their localization and morphology, were also exceptionally found at this time (Fig. 5A). In the 24-week diabetic retina, most of the deep invaginations of the photoreceptors contained degenerating

Fig. 4. An electron microphotograph showing part of a photoreceptor column delineated with the scleral radial process (rp) of a Müller cell. An apoptotic photoreceptor cell (white star) appears in between the normal photoreceptor cells (PC) at 8 weeks of diabetes. Scale bar represents $1 \mu \mathrm{m}$
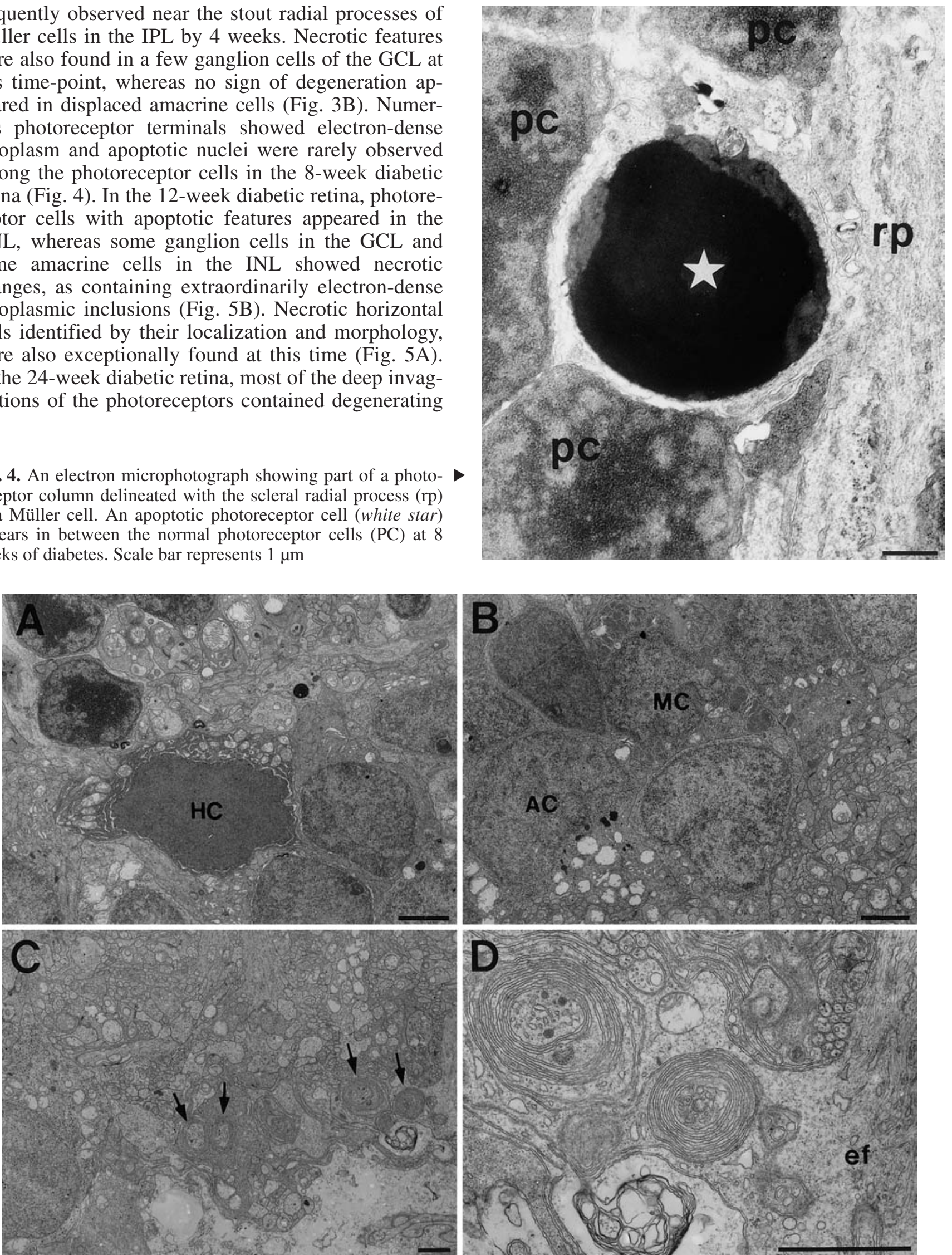

Fig. 5A-D. Electron microphotographs show parts of the inner plexiform layer in diabetic retinas at 12 weeks $(\mathbf{A}, \mathbf{B})$, and part of the ganglion cell layer at 24 weeks $(\mathbf{C}, \mathbf{D})$. A necrotic horizontal cell (HC) in $\mathbf{A}$, and a degenerative amacrine cell (AC)

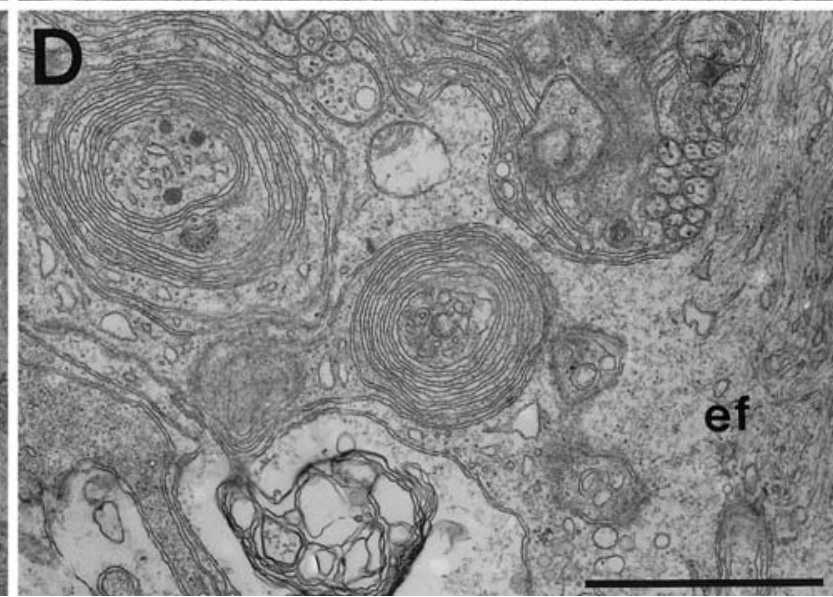

and a Müller cell (MC) packed with glycogen inclusion appeared in $\mathbf{B}$. $\mathbf{C}$ and $\mathbf{D}$ show degenerated nerve terminals (arrows) tightly rolled by the vitreal radial process of a Müller cell (ef). Scale bars represent $1 \mu \mathrm{m}$ 

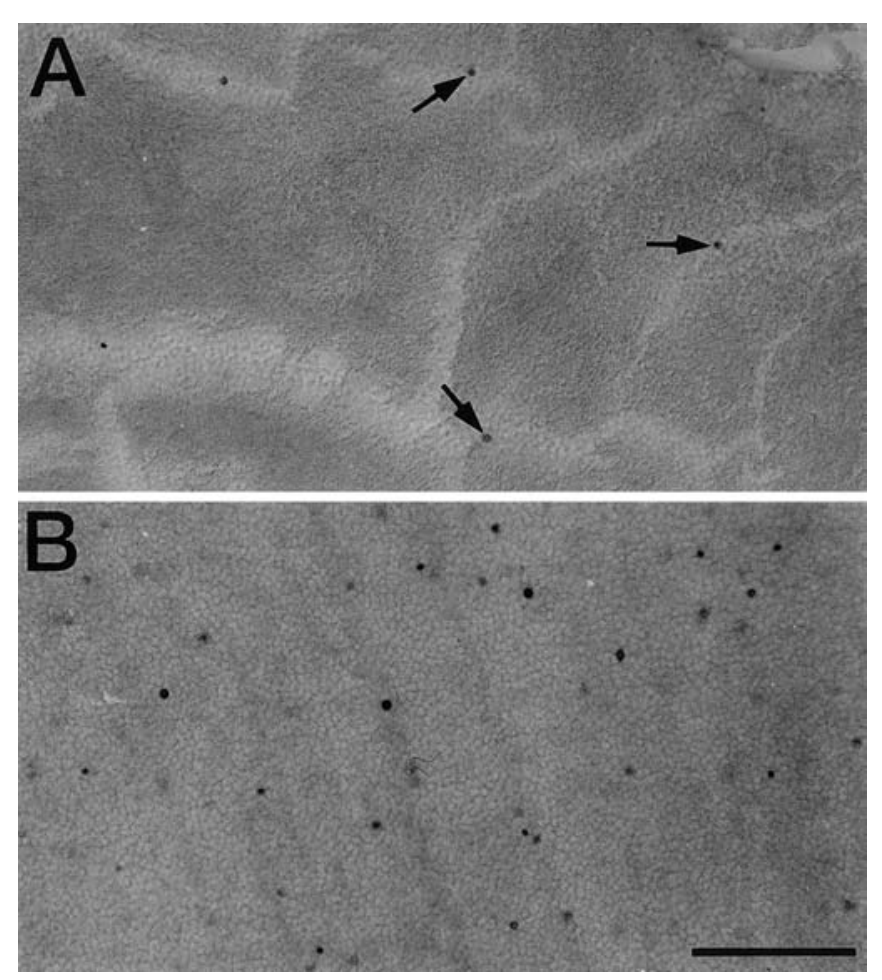

Fig. 6A, B. Light microphotographs being focused on the outer nuclear layer of parts of 4-week (A) and 12-week (B) diabetic whole mounted retinas processed for TUNEL staining. A low number of apoptotic nuclei (arrow) are present in $\mathbf{A}$, whereas about ten times more apoptotic nuclei are present in $\mathbf{B}$. Scale bar represents $100 \mu \mathrm{m}$
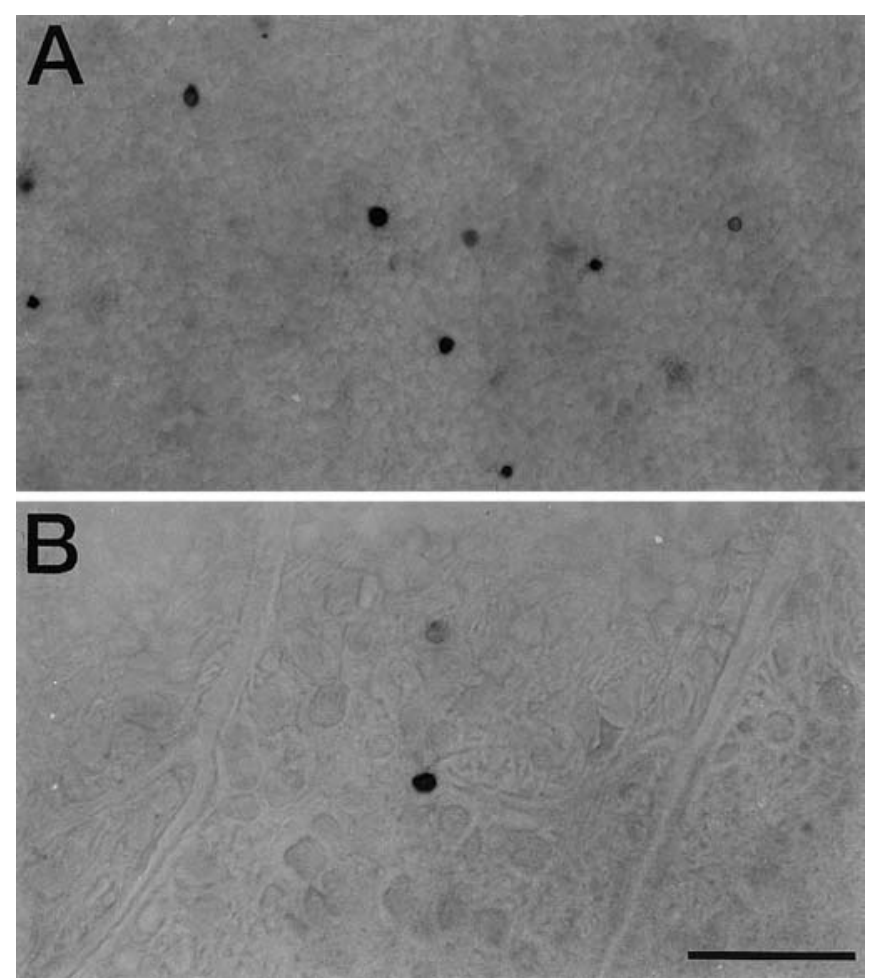

Fig. 7A, B. Light microphotographs focused on the outer nuclear layer (A) and the ganglion cell layer $(\mathbf{B})$ of 12-week diabetic whole mounted retina processed for TUNEL staining. Note that a few TUNEL positive nuclei also appear in the ganglion cell layer. Scale bar represents $50 \mu \mathrm{m}$ post-synaptic processes. In lamina five of the IPL, near the degenerating ganglion cells, the nerve terminals rolled spirally with the fine processes of Müller cells. Bipolar cell terminals with degenerating mitochondria were also remarkable at this time-point (Fig. 5C,D).

Apoptotic neuronal death detected by in situ TUNEL method. Throughout this experiment, TUNEL-positive apoptotic nuclei were observed in the ONL and the GCL. In 4-week diabetic retina, apoptotic nuclei were rarely observed in the middle of the ONL (Fig. 6A). The apoptotic photoreceptor cells were remarkable in 12-week and 24-week diabetic retinas and a few presumptive ganglion cells in the GCL were also TUNEL positive at these times (Figs. 6B, 7A,B).

\section{Discussion}

The maintenance of increased blood glucose concentrations above $300 \mathrm{mg} / \mathrm{dl}$ and a decrease in body weight were recorded for the streptozotocin-treated rats throughout the experiment. These measurements indicated that the Type 1 diabetes state, induced with a single dose of streptozotocin, is sufficient for tracing the early degeneration in the neural retina in ongoing diabetic retinopathy.

A transient increase in the thickness of the retinal layers was recorded in the inner retina at one week after diabetic onset. The main cause for this increase could be the breakdown of the blood-retinal barrier, especially the inner one, and the subsequent edematous swelling, in agreement with other studies $[15,16$, 17]. Therefore, a gradual decrease in the thickness of the inner retina, to well below that observed in the normal state, seems likely to arise from the decay of the neural components affected by the altered biochemical environment, including the glucose transport system $[18,19]$. A remarkable decrease in the thickness of the ONL is the first morphological finding, although physiological and clinical data had already provided evidence of changes in the photoreceptors under diabetic conditions [20,21].

The delayed occurrence of structural changes in the outer retina in our study has two possible explanations. One is the differences in energy sources between the inner and outer retinas. The outer retina is fed by the choriocapillaries, of a fenestrated type, located in the innermost part of the choroids [5, 22]. With these characteristics, the outer blood-retinal barrier requires the pigment epithelium to be tightly bound by tight junctions to protect the neural retina from the open circulation in the choroid. The pigment epithelium, in turn, not only has a specific glucose transporter, but also has its own growth factor, both of which are vital to the maintenance of homeostasis in the photoreceptor cells $[18,23]$. The other possibility arises from the hypothesis that the substances produced by other retinal neu- 
rons in response to diabetic injury, such as free radicals, react directly secondarily on the photoreceptor cells, ultimately leading to cell death. Of these free radicals, nitric oxide is a strong candidate for the role of an agent of inducible cytotoxicity, as photoreceptors contain a huge amount of cGMP, the final product of soluble guanyl cyclase, which is activated by nitric oxide [24]. It is possible that both these proposed mechanisms function in concert, because the changes in the ONL constitute a massive event.

The post-synaptic processes of the ribbon synapses in the photoreceptor terminals that show neurodegenerative changes throughout the stages of diabetes belong to horizontal cells. It is identified by their location in the deep invaginations of the photoreceptors [25]. Processes of some horizontal cells are located near the capillaries of the OPL, encompassing the basement membrane of the capillaries [26]. This characteristic of the horizontal cells can make them sensitive to any minute alteration in the capillaries, which are the deepest and the finest in the retinal vasculature [4]. The degenerative processes in the IPL are found mainly near the radial processes of the Müller cells, which constitute one component of the inner bloodretinal barrier in the INL [22]. Degenerative profiles at the neuronal level occur time sequentially, in proportion to their distance from the vasculature. These findings could explain that retinal neurons in the different spatial and temporal contexts are differentially sensitive to diabetic conditions, and this differential sensitivity correlates closely with the environment of the vasculature in the retina.

Both apoptosis and necrosis occur in the degeneration changes of neural retina in ongoing diabetic retinopathy in our study. Neurons undergoing necrotic degeneration were predominantly located in the inner retina, and appeared time sequentially. Therefore, necrosis in some neurons of the inner retina during diabetes is interpreted to be an inevitable primary effect of impaired glucose metabolism, which is consistent with in vitro glucose deprivation studies of the brain and the retina $[27,28]$. It is, however, not a cascade episode. In contrast, neural apoptosis occurred only in the ONL in our study. Apoptotic cell death is initiated in a very few photoreceptors about one month after the onset of diabetes, and accelerates from three months onwards. Thus, the main causal factor for the apoptosis in the ONL is not likely to be directly associated with altered glucose metabolism and consequent altered vascular conditions, as supported by another report [15, 29]. They describe that the main glucose transporter in the retina, GLT-1, is much more strongly expressed in the pigment epithelium than in the neural retina or its microvessels. GLT-1 in the epithelium is less affected or completely unaffected by diabetes. However, whether the regulatory mode of the GLT-1 in the neural retina in response to diabetes is upregulation [29] or downregulation $[18,30]$, is still controversial.
We detected apoptotic death of photoreceptors by the TUNEL method which was clearly distinguishable with electron microscopy. Earlier signs of visual dysfunction in patients with diabetic retinopathy were described as abnormal colour and contrast sensitivity evidenced by hue discrimination and electrophysiological methods [21, 31, 32, 33]. Regarding these results correlated with photoreceptor deficits, the remarkable appearance of apoptotic photoreceptors suggests that visual dysfunction in diabetic retinopathy may be initiated from the loss of the photoreceptors. However, there is a big controversy on which type of neurons undergoes apoptotic death in the diabetic retinas. Neural apoptosis in the ganglion cell layer has already been reported on the whole-mounted diabetic rat retinas [12]. Moreover, the neural apoptosis in diabetic retina has been suggested to be reversed by insulin therapy [12]. In our study, only a few presumptive ganglion cells showed apoptotic death at 12-week diabetic retina, when the appearance of apoptotic photoreceptors reaches a peak. This could be due to earlier necrotic changes of the ganglion cells. Judging from such different findings, there might be an unidentified mechanism modulating the neuronal apoptosis in the diabetic retina.

The glial reaction in both experimental and human diabetic retinas has been widely discussed [10, 34]. In this ultrastructural investigation, some end feet of Müller cells show a phagocytic feature. In the normal retina, debris of neurons in the inner retina are engulfed and digested by microglial cells, whereas photoreceptor debris are incorporated by pigment epithelial cells $[35,36]$. Microglial cells in the diabetic retina showed shape changes before the leakage of the blood-retinal barrier indicating their functional activation [37]. Hypertrophied microglial cells showed positional changes from the NFL and GCL to the IPL and rarely the OPL, and they increase in number [19]. This pattern of microglial activation is similar to what we observed immunohistochemically with an antibody against a lectin isoform. Based on these results and considering that the phagocytic character of Müller cells manifested only in the NFL, it is highly likely that the debris from the delayed degeneration of nerve fibers is cleared by activated Müller end feet. This proposition remains equivocal, even though in vitro studies have reported a phagocytic function for Müller cells $[38,39]$, and ultrastructural studies an increased lysosomal content in the Müller cells of streptozotocin-induced diabetic rats [40, 41].

The two findings, a severe loss of photoreceptors and the phagocytic feature of Müller end feet, could provide the morphological basis for the proposition that visual loss in diabetic retinopathy is initiated by neural dysfunction earlier than microangiopathy. Progression of diabetic retinopathy is considerably prevented by aminoguanidine [42]. It is also clarified that the initial phase of diabetic retinopathy is through non aminoguanidine-inhibited mechanism [42]. In con- 
trast, aminoguanidine has been suggested to act on restoring the number of nNOS-containing neurons in the short-term and long-term diabetic retinas, as mediated by the inhibition of AGE formation [43]. We suggest that future studies should be directed towards characterizing the mechanisms inducing the apoptosis of photoreceptors and ganglion cells. This could further the prevention of early neuronal degeneration in ongoing diabetic retinopathy.

Acknowledgements. The authors thank Mrs. Hee-Duck Rho and Hong-Lim Kim for their excellent technical assistance. This study was supported by a grant (R04-2000-000-00037-0) from the Basic Research Program of the Korea Science and Engineering Foundation.

\section{References}

1. King GL, Shiba T, Oliver J et al. (1994) Cellular and molecular abnormalities in the vascular endothelium of diabetes mellitus. Ann Rev Med 45:179-188

2. Vlassara H, Bucala R, Striker L (1994) Pathogenic effects of AGE's: biochemical, biologic, and clinical implications for diabetes and aging. Lab Invest 70:138-151

3. Lorenzi M, Gerhardinger C (2001) Early cellular and molecular changes induced by diabetes in the retina. Diabetologia 44:791-804

4. Gariano RF, Iruela-Arispe ML, Hendrickson AE (1994) Vascular development in primate retina: comparison of laminar plexus formation in monkey and human. Invest Ophthalmol Vis Sci 35:3442-3455

5. Essner E, Gordon SR (1983) Observation on the permeability of the choriocapillaries of the eye. Cell Tissue Res 231:571-577

6. Newman E, Reichenbach A (1996) The Muller cell: a functional element of the retina. TINS 19:307-311

7. Daley M, Watzke R, Riddle M (1987) Early loss of bluesensitive color vision in patients with type I diabetes. Diabetes Care 10:777-781

8. Sokol S, Moskowitz A, Skarf B (1985) Contrast sensitivity in diabetes with and without background retinopathy. Arch Ophthalmol 103:51-54

9. Ishikawa A, Ishiguro S, Tamai M (1996) Changes in GABA metabolism in streptozotocin-induced diabetic rat retinas. Curr Eye Res 15:63-71

10. Lieth E, Barber AJ, Xu B et al. (1998) Glial reactivity and impaired glutamate metabolism in short-term experimental diabetic retinopathy. Diabetes 47:815-820

11. Coupland SG (1987) Comparison of oscillatory potential and pattern electroretinogram measures in diabetic retinopathy. Doc Ophthalmol 66:207-218

12. Barber AJ, Lieth E, Khin SA et al. (1998) Neural apoptosis in the retina during experimental and human diabetes: Early onset and effect of insulin. J Clin Invest 102:783-791

13. Luo X, Lambrou GN, Sahel JA et al. (2001) Hypoglycemia induces general neuronal death, whereas hypoxia and glutamate transport blockade lead to selective retinal ganglion cell death in vitro. Invest Ophthalmol Vis Sci 42:2695-2705

14. Vorwerk CK, Kreutz MR, Bockers TM et al. (1999) Susceptibility of retinal ganglion cells to excitotoxicity depends on soma size and retinal eccentricity. Curr Eye Res 19:59-65

15. Antonetti DA, Barber AJ, Khin S et al. (1998) Vascular permeability in experimental diabetes is associated with reduced endothelial occludin content: vascular endothelial growth factor decreases occludin in retinal endothelial cells. Diabetes 47:1953-1959

16. Carmo AD, Ramos P, Reis A, Proenca R, Cunha-Vaz JG (1998) Breakdown of the inner and outer blood retinal barrier in streptozotocin-induced diabetes. Exp Eye Res 67:569-575

17. Barber AJ, Antonetti DA, Gardner TW et al. (2000) Altered expression of retinal occludin and glial fibrillary acidic protein in experimental diabetes. Invest Ophthalmol Vis Sci 41:3561-3568

18. Kumagai AK, Glasgow BJ, Pardridge WM (1994) GLUT1 glucose transporter expression in the diabetic and nondiabetic human eye. Invest Ophthalmol Vis Sci 35:28872894

19. Zeng X-X, Ng Y-K, Ling E-A (2000) Neuronal and microglial response in the retina of streptozotocin-induced diabetic rats. Vis Neurosci 17:463-471

20. Greenstein V, Sarter B, Hood D et al. (1990) Hue discrimination and $\mathrm{S}$ cone pathway sensitivity loss in diabetes. Invest Ophthalmol Vis Sci 31:1008-1014

21. Holopigian K, Greenstein VC, Seiple W et al. (1997) Evidence for photoreceptor changes in patients with diabetic retinopathy. Invest Ophthalmol Vis Sci 38:2355-2365

22. Holländer H, Makarov F, Dreher Z et al. (1991) Structure of the macroglia of the retina: sharing and division of labour between astrocytes and Müller cells. J Comp Neurol 313:587-603

23. Cao W, Tombran-Tink J, Chen W et al. (1999) Pigment epithelium-derived factor protects cultured retinal neurons against hydrogen peroxide-induced cell death. J Neurosci Res 57:789-800

24. Gotzes S, Vente J de, Müller F (1998) Nitric oxide modulates cGMP levels in neurons of the inner and outer retina in opposite ways. Vis Neurosci 15:945-955

25. Dowling JE (1987) Wiring of the retina. In: Dowling JE (ed.) The retina: an approachable part of the brain. The Belknap Press of Harvard University Press, Cambridge, pp 42-80

26. Knabe W, Kuhn HJ (2000) Capillary-contacting horizontal cells in the tree shrew Tupaia belangeri belong to the mammalian type A. Cell Tissue Res 299:307-311

27. Joo CK, Choi JS, Ko HW et al. (1999) Necrosis and apoptosis after retinal ischemia: involvement of NMDA-mediated excitotoxicity and p53. Invest Ophthalmol Vis Sci 40:713-720

28. Laake JH, Haung FM, Wieloch T et al. (1999) A simple in vitro model of ischemia based on hippocampal slice cultures and propidium iodide fluorescence. Brain Res Brain Protoc 4:173-184

29. Badr GA, Tang J, Ismail-Beigi F et al. (2000) Diabetes downregulates GLUT1 expression in the retina and its microvessels but not in the cerebral cortex or its microvessels. Diabetes 49:1016-1021

30. Kumagai AK, Vinores SA, Pardridge WM (1996) Pathological upregulation of inner blood-retinal barrier Glut1 glucose transporter expression in diabetes mellitus. Brain Res 706:313-317

31. Greenstein V, Sarter B, Hood D et al. (1990) Hue discrimination and $\mathrm{S}$ cone pathway sensitivity in early diabetic retinopathy. Invest Ophthalmol Vis Sci 31:1008-1014

32. Hardy KJ, Fisher C, Heath P et al. (1995) Comparison of colour discrimination and electroretinography in evaluation of visual pathway dysfunction in aretinopathic IDDM patients. Br J Ophthalmol 79:35-37

33. Yamamoto S, Kamiyama M, Nitta K et al. (1996) Selective reduction of the $\mathrm{S}$ cone electroretinogram in diabetes. $\mathrm{Br} \mathrm{J}$ Ophthalmol 80:973-975

34. Mizutani M, Gerhardinger C, Lorenzi M (1998) Müller cell changes in human diabetic retinopathy. Diabetes 47:445449 
35. Ashwell K (1989) Development of microglia in the albino rabbit retina. J Comp Neurol 287:286-301

36. Nguyen-Legros J, Hicks D (2000) Renewal of photoreceptor outer segments and their phagocytosis by the retinal pigment epithelium. Int Rev Cytol 196:245-313

37. Rungger-Brändle E, Dosso AA, Leuenberger PM (2000) Glial reactivity, an early feature of diabetic retinopathy. Invest Ophthalmol Vis Sci 41:1971-1980

38. Wagner EC, Raymond PA (1991) Müller cells of the goldfish retina are phagocytic in vitro but not in vivo. Exp Eye Res 53:583-589

39. Stolzenberg JU, Haas J, Hartig W et al. (1992) Phagocytosis of latex beads by rabbit Müller (glial) cells in vitro. J Hirnforsch 33:557-564
40. Hori S, Nishida T, Mukai N (1980) Ultrastructural studies on lysosomes in retinal Müller cells of streptozotocin-diabetic rats. Invest Ophthalmol Vis Sci 19:1295-1300

41. Hori S, Mukai N (1980) Ultrastructural lesions of retinal pericapillary Müller cells in streptozotocin-induced diabetic rats. Graefes Arch Clin Exp Ophthalmol 213:1-9

42. Hammes HP, Syed S, Uhlmann M et al. (1995) Aminoguanidine does not inhibit the initial phase of experimental diabetic retinopathy in rats. Diabetologia 38:269-273

43. Roufail E, Soulis T, Boel E et al. (1998) Depletion of nitric oxide synthase-containing neurons in the diabetic retina: reversal by aminoguanidine. Diabetologia 41: 1419-1425 